\title{
Influences on the Simon effect of prior practice with spatially incompatible mappings: Transfer within and between horizontal and vertical dimensions
}

\author{
Kim-PhuONG L. Vu \\ California State University, Long Beach, California
}

\begin{abstract}
The Simon effect refers to the fact that for tasks in which stimulus location is irrelevant and a nonspatial attribute is relevant, responses are typically faster when stimulus and response locations correspond than when they do not. Two experiments examined the influence of prior practice with an incompatible relevant spatial mapping on the Simon effect as a function of the dimension (vertical or horizontal) along which the stimuli and responses varied in practice and transfer sessions. With 72 practice trials, the Simon effect in the transfer session was eliminated only when the spatial dimension was horizontal for both practice and transfer. With 600 practice trials, the Simon effect was eliminated for all combinations of practice and transfer dimensions, with noncorresponding responses showing an advantage when the dimension was horizontal for both practice and transfer. Within-dimension transfer effects for the horizontal dimension after a small amount of practice can be attributed to reactivation of specific stimulus-response associations defined for the practice task. However, the between-dimension transfer effects evident after a larger amount of practice cannot be explained in this manner and suggest that the subjects acquired a general procedure of responding opposite to the stimulus location.
\end{abstract}

Stimulus-response compatibility (SRC) refers to the fact that performance is better with certain mappings of stimuli to responses than with others (Proctor \& Vu, 2006). When left and right stimulus locations are mapped to left and right keypresses, the mapping of a left stimulus to a left response and a right stimulus to a right response yields better performance than does the alternative mapping. Spatial compatibility effects also occur when stimulus location is irrelevant and the relevant information is conveyed by a nonspatial stimulus attribute (e.g., color or shape), a phenomenon known as the Simon effect (Lu \& Proctor, 1995; Simon, 1990; Simon \& Rudell, 1967). Similar effects occur in two-choice tasks when the stimuli and responses are arrayed vertically (Nicoletti \& Umiltà, 1985; Vu, Pellicano, \& Proctor, 2005; Vu, Proctor, \& Pick, 2000).

\section{Accounts of SRC and Simon Effects}

Accounts of spatial SRC effects, including SRC proper and the Simon effect, have focused mainly on spatial coding, because compatibility effects depend on the relative positions of the stimuli and the responses (e.g., Nicoletti, Anzola, Luppino, Rizzolatti, \& Umiltà, 1982; Roswarski \& Proctor, 2000). All models include an intentional translation process that influences the speed of response selection for SRC proper (e.g., Duncan, 1977; Proctor \& Reeve, 1985, 1986). Duncan proposed that SRC effects occur because $\mathrm{S}-\mathrm{R}$ translation is accomplished by way of an identity rule (respond at the location that is the same as that of the stimulus) when the mapping is spatially compatible but by a less efficient respond-opposite rule (respond at the location that is opposite that of the stimulus) when it is incompatible. Although all models of SRC effects include a translational component, most recent models attribute SRC effects, in part, to a second process: direct (or automatic) activation of the corresponding response (e.g., Hommel, 1997; Kornblum, Hasbroucq, \& Osman, 1990).

According to two-process models of compatibility effects, the corresponding response is activated regardless of whether stimulus location is relevant or irrelevant. For SRC proper, this activation is often assumed to facilitate responding when the mapping is compatible but to interfere when it is incompatible. For the Simon effect, responding is faster when the response assigned to the relevant stimulus attribute is the same as that activated by the stimulus position but slower when it is not, due to conflict between activated and assigned response codes. The Simon effect is typically attributed to automatic activation alone, whereas differences in translation efficiencies of the type mentioned above are presumed to contribute as well to the effect of SRC proper (e.g., Kornblum et al., 1990).

Although the roles of automatic activation and intentional translation in SRC and Simon effects can be dis-

K.-P. L.Vu, kvu8@csulb.edu 
cussed in terms of two different processes, these processes can also be depicted in terms of short-term (or taskdefined) and long-term associations. Accounts of SRC effects emphasizing strengths of S-R associations can be traced back to Donders (1868/1969), who first demonstrated such effects, and Fitts (1964), who formalized the study of compatibility effects (see Proctor \& Vu, 2002). Both Donders and Fitts attributed the benefit for the compatible mapping, at least in part, to responding with natural tendencies established through habit or training (i.e., long-term associations). The contributions of taskdefined and long-term associations have also been emphasized in more recent characterizations of compatibility effects (e.g., Barber \& O’Leary, 1997; Stoffer \& Umiltà, 1997; Zorzi \& Umiltà, 1995). In a choice reaction task, the instructions define temporary associations between the relevant stimulus values and the responses for the task, but subjects come to the experiment with long-term associations between corresponding stimulus and response locations. In this view, direct activation of the corresponding response via long-term associations is solely responsible for producing the Simon effect, whereas SRC proper is a function of both this activation and relative efficiencies of the task-defined S-R associations.

\section{Transfer of Task-Defined Spatial Associations to the Simon Task}

An implication of the typical depiction of SRC and Simon effects is that the associations defined for the task are maintained only as long as the task is in effect and should cease to influence performance when they are no longer relevant. Yet several recent studies have shown that this is not the case. Proctor and Lu (1999) had subjects perform a two-choice SRC-proper task using an incompatible spatial mapping for more than 900 trials, spread over 3 days, and then transferred them to a Simon task on the 4th day. The Simon effect reversed, showing better performance when stimulus and response locations did not correspond than when they did. Thus, although stimulus location was no longer relevant in the transfer task, the noncorresponding response continued to be activated at stimulus onset, instead of the corresponding response. Tagliabue, Zorzi, Umiltà, and Bassignani (2000) showed that as few as 72 trials of practice with a spatially incompatible mapping were sufficient to eliminate the Simon effect when the Simon task was performed 5 min after the practice session. Moreover, they found that when the Simon task was performed after a week delay, the Simon effect reversed to favor the noncorresponding response. Tagliabue et al. (2000) attributed this reversal after a week delay to consolidation of the task-defined associations.

Transfer designs allow not only demonstration of the continued influence of the practiced S-R mapping, but also evaluation of what is specifically learned during practice (e.g., Healy, Wohldmann, \& Bourne, 2005; Pashler \& Baylis, 1991; Proctor \& Dutta, 1995). One issue of general interest is the specificity of learning. Tagliabue, Zorzi, and Umiltà (2002) evaluated whether the procedures acquired from practice with the incompatible spatial mapping are modality specific or more general. They had subjects practice for 72 trials with an incompatible mapping of left and right tones to keypress responses, and after delays of $5 \mathrm{~min}, 1$ day, or 1 week, the subjects performed a visual Simon task. The Simon effect was eliminated at all delays, but it did not reverse at the 1-week delay, unlike when the stimulus modality was visual in both practice and transfer (Tagliabue et al., 2000). However, a betweenstudy comparison of the week-delayed Simon data showed no significant difference in the visual Simon effect as a function of whether the practice modality was visual or auditory. This led Tagliabue et al. (2002) to conclude that the elimination or reversal of the Simon effect is due to a spatial remapping that is not modality specific.

Because the comparison made by Tagliabue et al. (2002) between the effects of auditory and visual practice was between different studies and of low power, Vu, Proctor, and Urcuioli (2003) conducted an experiment in which they replicated the 1-week delay conditions from Tagliabue et al.'s (2002; Tagliabue et al., 2000) studies within a single experiment. The Simon effect obtained with visual stimuli was reversed when the practice task was visual but was eliminated only when the practice task was auditory, indicating that the apparent difference between Tagliabue et al.'s (2002; Tagliabue et al., 2000) studies is real and that there are both modality-specific and more general components to the transfer effect.

\section{Specific S-R Associations Versus Systematic S-R Relations}

Tagliabue et al. (2002; Tagliabue et al., 2000) concluded that the elimination (or reversal) of the visual Simon effect after practice with an incompatible spatial mapping is due to the spatial associations for the practice task's remaining active during the subsequent Simon task. Although their findings and those of $\mathrm{Vu}$ et al. (2003) indicate that these associations, or procedures, are, at least in part, more general than the stimulus modality used for practice, the associations may still be specific to the left-right spatial codes generated in practice and transfer. $\mathrm{S}-\mathrm{R}$ sets that vary along different dimensions in practice and transfer can be used to evaluate whether the learning of location relations that occurs involves associations specific to the spatial codes generated in practice or a general procedure that transfers to locations on an orthogonal spatial dimension. Transfer should be restricted to the same dimension if the transfer effect is due either to continued activation of the links between individual stimulus locations and their assigned responses (Tagliabue et al., 2002) or to retrieval of episodes of making the noncorresponding response to a stimulus with a given spatial code (e.g., Logan, 1988). A finding of transfer across different practice and stimulus dimensions would suggest that the source of the effect is a respond-opposite procedure acquired during practice (Duncan, 1977; Rosenbloom, 1986).

Two experiments were conducted to examine these predictions. In both experiments, subjects practiced with an incompatible mapping of two visual stimulus locations to two keypress responses and then were transferred to a Simon task in which stimulus color was relevant and location irrelevant. Orientation of the $\mathrm{S}-\mathrm{R}$ sets during prac- 
tice was either horizontal or vertical, as was orientation of the S-R sets during transfer. With this design, withindimension and cross-dimension transfer effects could be examined for both horizontal and vertical Simon tasks.

In Experiment 1, the subjects practiced for 72 trials with an incompatible spatial mapping and then performed the Simon transfer task. If the transfer effect is due primarily to specific $\mathrm{S}-\mathrm{R}$ associations, the effect should be evident only for conditions in which the spatial dimension in the transfer session is the same as that in the practice session. However, if the transfer effect is due to a more general respond-opposite procedure, the effect likely would be evident between dimensions, as well as within. The transfer test was conducted at a delay of 5 min or 1 week, to assess whether the basis for the transfer effect shifts from one of activation/retrieval of specific S-R links at a short delay to one of rule-based procedures over the intervening week.

Experiment 2 was similar to Experiment 1, except that only the 5-min delay was used and, more important, subjects performed 600 trials of practice with the incompatible spatial mapping, instead of 72 trials. With more practice, the likelihood increases that a general respondopposite procedure will develop (Rosenbloom, 1986), causing transfer to occur between $\mathrm{S}-\mathrm{R}$ dimensions, as well as within.

\section{EXPERIMENT 1}

In Experiment 1, the influence of 72 trials of practice with an incompatible mapping of horizontal or vertical S-R locations on performance of a horizontal or vertical Simon task performed after a 5-min or a 1-week delay was examined. If the influence of the prior practice on the visual Simon task is due to persisting activation of the specific noncorresponding $\mathrm{S}-\mathrm{R}$ associations learned in practice or to the ease with which the noncorresponding response can be retrieved, transfer to the Simon task should occur only when the dimension along which the stimuli and responses vary is the same in both tasks. If transfer effects across different practice and transfer dimensions are obtained, the effects are most likely due to acquisition of a respond-opposite procedure that continues to be applied to the stimulus location in the Simon task even though location is nominally irrelevant.

\section{Method}

Subjects. One hundred ninety-two students were recruited from Purdue University's introductory psychology courses to participate for partial course credit. All the subjects reported having normal or corrected-to-normal vision. Ninety-six practiced with an incompatible mapping of horizontal stimuli and responses, and 96 with an incompatible mapping of vertical stimuli and responses. For the group that practiced with an incompatible mapping of horizontally arrayed S-R sets, 48 were transferred to a horizontal Simon task after a 5-min delay $(n=24)$ or after a 1 -week delay $(n=24)$, and 48 were transferred to a vertical Simon task after a 5-min delay $(n=$ $24)$ or after a 1 -week delay $(n=24)$. Similarly, for the group that practiced with an incompatible mapping of vertically arrayed S-R sets, 48 were transferred to a horizontal Simon task after a 5-min delay $(n=24)$ or after a 1 -week delay $(n=24)$, and 48 were transferred to a vertical Simon task after a 5-min delay $(n=24)$ or after a 1-week delay $(n=24)$.
Apparatus, Stimuli, and Procedure. The stimuli were presented on personal computers, with 14-in. VGA color monitors. Micro Experimental Laboratory (MEL Version 2.01) was used to control the experiment. The subjects sat directly in front of the monitor, at a viewing distance of approximately $60 \mathrm{~cm}$. The stimuli were white, red, or green solid circles (MEL color codes 15, 4, and 2, respectively) approximately $1.2 \mathrm{~cm}$, or $1.1^{\circ}$, in diameter. The stimuli were presented in left and right locations for the horizontal dimension and top and bottom locations for the vertical dimension, approximately $7 \mathrm{~cm}\left(6.65^{\circ}\right)$ from the center of the screen. Responses were made on the numeric pad of the keyboard by using the " 4 " and " 6 " keys (left and right keys) for the horizontal dimension and the " 8 " and "2" keys (top and bottom keys, aligned vertically) for the vertical dimension. The left index finger was used to press the first key in each pair, and the right index finger the second key.

For the practice session, the circles were white. For the horizontal practice task, the subjects were instructed to respond with a spatially incompatible mapping of left stimulus to right response and right stimulus to left response. For the vertical practice task, the subjects were instructed to respond to the top stimulus by pressing the bottom key and to the bottom stimulus by pressing the top key. The practice session consisted of 72 trials.

Half of the subjects were transferred to the Simon task after a 5-min delay, and half after a 1-week delay. For the transfer session, the subjects were told to respond to the color of the stimulus on each trial and to ignore its location. For the subjects in each orientation condition, the red stimulus was assigned to one response, and the green stimulus to the other response, with the assignment counterbalanced across subjects. All the subjects performed one block of the Simon trials, which consisted of 144 trials. The entire session lasted about $40 \mathrm{~min}$.

For all the conditions, the order in which the stimuli appeared was randomized. The subjects were instructed to respond as quickly and accurately as possible. Reaction time (RT) was measured from stimulus onset to the depression of a response key. The stimulus remained present for $1,500 \mathrm{msec}$ or until a response was made, and the intertrial interval was $1 \mathrm{sec}$. The trial terminated if the subjects did not respond within $1,500 \mathrm{msec}$. A $400-\mathrm{Hz}$ error tone was presented for $500 \mathrm{msec}$ for incorrect responses or terminated responses.

\section{Results}

The data were analyzed separately for the practice and the transfer sessions. The first 12 trials of each session were considered warm-up and were not included in the analyses.

Practice session. Two (practice dimension: horizontal or vertical) $\times 2$ (transfer dimension: horizontal or vertical) $\times 2$ (delay: 5 min or 1 week) ANOVAs were performed on RT and percentage of error (PE; see Table 1 for means). All the factors were between subjects. The main effect of practice dimension was significant for RT $[F(1,184)=$ $\left.21.46, M S_{\mathrm{e}}=5,272, p<.001\right]$ and $\operatorname{PE}[F(1,184)=5.01$, $\left.M S_{\mathrm{e}}=6.16, p=.026\right]$. RTs were shorter and errors tended to be fewer when the practice dimension was horizontal $(M=384 \mathrm{msec}$ and $2.24 \%)$ than when it was verti$\mathrm{cal}(M=433 \mathrm{msec}$ and $3.04 \%)$. The two-way interactions of practice dimension and transfer dimension were not significant for either measure $(F \mathbf{s}<1)$, indicating that the transfer dimension groups showed similar performance in the practice session prior to transfer to the Simon task. Moreover, there were no significant interactions of delay with any other factors $(F \mathrm{~s}<1)$.

Transfer session. Mean RTs and PEs for corresponding and noncorresponding trials were obtained in each condition in order to examine how the Simon effect is in- 
Table 1

Mean Reaction Times (RTs) and Percentages of Errors (PEs) in the Practice Sessions in Experiments 1 and 2 (With Standard Deviations)

\begin{tabular}{|c|c|c|c|c|}
\hline \multirow[b]{3}{*}{ Practice-Transfer Dimension } & \multicolumn{4}{|c|}{ Performance } \\
\hline & \multicolumn{2}{|c|}{ RT } & \multicolumn{2}{|c|}{$\mathrm{PE}$} \\
\hline & $M$ & $S D$ & $M$ & $S D$ \\
\hline \multicolumn{5}{|c|}{ Experiment 1: 5-Min Delay } \\
\hline Vertical-vertical & 422 & 88 & 3.21 & 2.5 \\
\hline Vertical-horizontal & 441 & 93 & 3.70 & 3.7 \\
\hline Horizontal-vertical & 391 & 70 & 2.30 & 2.4 \\
\hline Horizontal-horizontal & 405 & 65 & 2.37 & 2.4 \\
\hline \multicolumn{5}{|c|}{ Experiment 1: 1-Week Delay } \\
\hline Vertical-vertical & 435 & 72 & 2.65 & 2.3 \\
\hline Vertical-horizontal & 433 & 75 & 2.59 & 2.5 \\
\hline Horizontal-vertical & 366 & 53 & 1.88 & 1.2 \\
\hline Horizontal-horizontal & 374 & 57 & 2.39 & 2.3 \\
\hline \multicolumn{5}{|c|}{ Experiment 2} \\
\hline Vertical-vertical & 411 & 56 & 1.77 & 1.2 \\
\hline Vertical-horizontal & 390 & 62 & 1.66 & 1.2 \\
\hline Horizontal-vertical & 380 & 69 & 1.21 & 0.7 \\
\hline Horizontal-horizontal & 364 & 48 & 1.46 & 1.2 \\
\hline
\end{tabular}

fluenced by prior practice with an incompatible mapping. Separate 2 (practice dimension: horizontal or vertical) $\times$ 2 (transfer dimension: horizontal or vertical) $\times 2$ (correspondence: corresponding or noncorresponding) $\times 2$ (delay: 5 min or 1 week) ANOVAs were performed for RT and PE (see Table 2). For both measures, the main effects of correspondence were significant $[F \mathrm{~s}(1,184)=70.69$ and 19.22, $M S_{\mathrm{e}} \mathrm{s}=370$ and 4.89, respectively; $\left.p \mathrm{~s}<.001\right]$, with the overall Simon effect being $17 \mathrm{msec}$ and $1.01 \%$. For RT, correspondence interacted with transfer dimension $\left[F(1,184)=11.14, M S_{\mathrm{e}}=370, p=.001\right]$, indicating that the vertical Simon effect $(M D=23 \mathrm{msec})$ was larger than the horizontal Simon effect $(M D=10 \mathrm{msec})$. For both RT and PE, the main effect for delay was not significant $[F \mathrm{~s}(1,184)<2.02, p \mathrm{~s}>.16]$, and delay did not interact with any other factors $[F \mathrm{~s}(1,184)<1.85, p \mathrm{~s}>.18]$.

Most important, for both measures, the three-way interaction between correspondence, practice dimension, and transfer dimension was significant $[F \mathrm{~s}(1,184)=4.50$ and 5.01, $M S_{\mathrm{e}} \mathrm{s}=370$ and 4.89, respectively; $p \mathrm{~s}<.035$ ]. To evaluate the three-way interactions, separate ANOVAs were performed for the horizontal and the vertical Simon tasks. For the horizontal task, correspondence interacted significantly with practice dimension for RT $[F(1,94)=7.13$, $\left.M S_{\mathrm{e}}=373, p<.010\right]$ and almost so for PE $[F(1,94)=$ $\left.3.34, M S_{\mathrm{e}}=6.07, p=.071\right]$. The subjects who practiced with an incompatible horizontal mapping showed no Simon effect for RT or PE $\left(F_{\mathrm{S}}<1\right)$, consistent with the results of previous studies, but those who practiced with an incompatible vertical mapping did show a Simon effect for both measures $[F \mathrm{~s}(1,47)=19.29, p \mathrm{~s}<.001]$. For the vertical Simon task, the interaction of correspondence and practice dimension was not significant $[F<1$ for RT; $F(1,94)=$ $1.70, M S_{\mathrm{e}}=3.66, p=.20$, for PE]. The vertical Simon effect was significant for both RT and PE $[F \mathrm{~s}(1,47)>7.87$, $p \mathrm{~s}<.007$ ], regardless of whether prior practice was with an incompatible horizontal or an incompatible vertical mapping. No other effects were significant.

\section{Discussion}

The horizontal Simon effect was eliminated after the subjects had practiced for 72 trials with an incompatible mapping of horizontal locations, a finding that is in agreement with the previous results of Tagliabue et al. (2000) and $\mathrm{Vu}$ et al. (2003). However, this finding did not generalize to the situation in which the $\mathrm{S}-\mathrm{R}$ sets varied along the vertical dimension in both the practice and the transfer sessions. It is surprising that the vertical Simon effect was not eliminated by prior practice with an incompatible mapping along the same dimension, because the primary difference between this situation and the within-dimension transfer for the horizontal Simon task, for which the Simon was eliminated, is one of orientation.

The lack of a transfer effect within the vertical dimension may be a consequence of the fact that the mechanisms that cause the vertical Simon effect are not identical to those that cause the horizontal Simon effect. The vertical and horizontal Simon effects show different temporal properties (Proctor, Vu, \& Nicoletti, 2003; Wiegand

Table 2

Mean Reaction Times (RTs) and Percentages of Errors (PEs) (With Standard Deviations) and Simon Effects in Experiment 1 As a Function of Correspondence, Practice-Transfer Dimension, and Delay

Correspondence

\begin{tabular}{|c|c|c|c|c|c|c|c|c|c|c|}
\hline \multirow{4}{*}{$\begin{array}{c}\text { Practice-Transfer } \\
\text { Dimension }\end{array}$} & \multicolumn{8}{|c|}{ Correspondence } & & \\
\hline & \multicolumn{4}{|c|}{ Corresponding } & \multicolumn{4}{|c|}{ Noncorresponding } & & \\
\hline & \multicolumn{2}{|c|}{ RT } & \multicolumn{2}{|c|}{$\mathrm{PE}$} & \multicolumn{2}{|c|}{ RT } & \multicolumn{2}{|c|}{$\mathrm{PE}$} & \multicolumn{2}{|c|}{$\underline{\text { Simon Effect }}$} \\
\hline & $M$ & $\overline{S D}$ & $M$ & $\overline{S D}$ & $M$ & $\overline{S D}$ & $M$ & $\overline{S D}$ & RT & $\mathrm{PE}$ \\
\hline \multicolumn{11}{|c|}{ 5-Min Delay } \\
\hline Vertical-vertical & 456 & 63 & 1.81 & 2.4 & 481 & 77 & 3.01 & 1.9 & 25 & 1.20 \\
\hline Vertical-horizontal & 457 & 48 & 1.89 & 3.2 & 472 & 55 & 3.12 & 3.0 & 15 & 1.23 \\
\hline Horizontal-vertical & 481 & 57 & 1.25 & 2.1 & 509 & 52 & 3.29 & 2.8 & 28 & 2.04 \\
\hline Horizontal-horizontal & 466 & 56 & 1.52 & 1.5 & 470 & 56 & 2.24 & 2.9 & 4 & 0.72 \\
\hline \multicolumn{11}{|c|}{ 1-Week Delay } \\
\hline Vertical-vertical & 442 & 41 & 1.28 & 1.4 & 462 & 42 & 2.19 & 2.2 & 20 & 0.91 \\
\hline Vertical-horizontal & 454 & 70 & 1.61 & 1.8 & 474 & 56 & 2.87 & 2.8 & 20 & 1.26 \\
\hline Horizontal-vertical & 470 & 63 & 1.33 & 1.8 & 489 & 59 & 2.85 & 2.4 & 19 & 1.52 \\
\hline Horizontal-horizontal & 454 & 54 & 2.91 & 2.5 & 456 & 50 & 2.09 & 2.5 & 2 & -0.82 \\
\hline
\end{tabular}


$\&$ Wascher, 2005) and onsets of the lateralized readiness potential (a psychophysiological indicator of response activation; see Wiegand \& Wascher, 2005). On the basis of these findings, Wiegand and Wascher concluded that

there are two dissociable mechanisms involved in generating the Simon effect: a visuomotor facilitation of same sided responses, which leads to a transient Simon effect (only observed for horizontal S-R relations) and a cognitive interference of codes leading to a sustained Simon effect. (p. 463)

Wiegand and Wascher noted that the left-right hand distinction is congruent with the left-right spatial distinction for the horizontal Simon task, but not with the top-bottom spatial distinction for the vertical Simon task. Consequently, they proposed that the transient component of the horizontal Simon effect "appears to be related to spatial anatomical mapping and might be based on automatic activation of the corresponding response (hand)" (p. 463). When stimulus location is relevant, as in the practice session of the present experiment, the development of noncorresponding S-R associations may reduce the transient component for the horizontal dimension in the Simon transfer session, leading to the elimination of the horizontal Simon effect.

When the S-R dimensions in the practice and transfer sessions were different, there was no transfer of the prior incompatible mapping to the Simon task. Thus, for the horizontal Simon task, transfer of the practice mapping occurred only when the practice task also involved the horizontal dimension. This pattern of results provides no evidence for transfer of a respond-opposite procedure. The absence of a cross-dimensional transfer effect from practice with the horizontal dimension to the vertical Simon task is less informative, because the vertical Simon effect was evident even when practice involved the vertical dimension. Thus, the within- and between-dimension comparison for the vertical Simon task does not speak to the issue of specificity of transfer.

With a 1-week delay between practice and transfer sessions, the influence of the prior incompatible mapping did not differ significantly from that obtained when the delay was only $5 \mathrm{~min}$. This finding is in agreement with those of Vu et al. (2003) and their conclusion that consolidation does not act to increase the effect of the practiced incompatible mapping over a 1-week delay. That the transfer effect for the horizontal practice and horizontal transfer condition persists over the 1-week delay, even though not increasing, could be a result of the reinstatement of the same motoric procedures in the transfer session as in the practice session (e.g., Healy et al., 2005).

\section{EXPERIMENT 2}

As has been noted, Proctor and Lu (1999) showed that the Simon effect was reversed to favor noncorresponding responses after subjects had performed 948 incompatibly mapped trials over 3 days and had transferred to the Simon task on the 4th day. The reversed effect in their study averaged $-20 \mathrm{msec}(-14$ and $-25 \mathrm{msec}$ in their Experiments 2 and 3, respectively), which was of an ab- solute magnitude similar to the $21-\mathrm{msec}$ positive Simon effect that Proctor and $\mathrm{Lu}$ obtained after practicing with a compatible location mapping. Thus, Proctor and Lu's findings suggest that the amount of transfer increases as the number of trials on the practice task increases.

Therefore, in Experiment 2, the number of practice trials was increased from 72 to 600 . A larger transfer effect should be evident after 600 practice trials, with one possible reason being that the strength of the specific noncorresponding $\mathrm{S}-\mathrm{R}$ associations, relative to that of the long-term corresponding associations, would be increased by the additional practice on the spatial task. Another possible reason would be that the extra practice would enable the subjects to represent response selection in the practice task as a respondopposite procedure, which would then be transferred to the Simon task. Because Experiment 1 had shown no effect of delay, only a 5-min delay was used in Experiment 2. Control conditions, in which no prior practice was performed, were added in order to provide baselines for evaluating whether the Simon effect was reduced significantly for the dimension combinations that showed no reduction with the lesser amount of practice in Experiment 1.

As in Experiment 1, both horizontal and vertical S-R sets were used to evaluate the specificity of transfer. If increasing practice with the incompatible location mapping influences only the horizontal Simon effect, and not the vertical Simon effect, the transfer is specific to processes involved in the horizontal task. If the increased practice results in larger transfer effects for both the horizontal and the vertical S-R sets when they are the same in the practice and transfer sessions, but not when they are different, this would suggest that the lack of the transfer effect for the vertical-practice-vertical-transfer condition in Experiment 1 was due to the noncorresponding associations being of insufficient strength. If the Simon effect is also eliminated in the between-dimension transfer conditions, the implication would be that the additional practice trials allow development of the respond-opposite procedure, which transfers to the Simon task.

\section{Method}

Ninety-six new undergraduates from the same subject pool as that in the previous experiment participated; 32 were assigned to each of three practice conditions: horizontal-incompatible mapping, vertical-incompatible mapping, and control (no prior practice with an incompatible mapping). Within each practice condition, 16 subjects performed a horizontal Simon task, and 16 a vertical Simon task. For the conditions in which the subjects practiced with an incompatible mapping, the number of trials in the practice session was 600 , performed in two blocks of 300, with a 5-min break in between. Following the second practice block, the subjects performed the transfer condition after a 5-min delay. Otherwise, the apparatus, stimuli, and procedure were similar to those used in Experiment 1. The experimental session lasted about $20 \mathrm{~min}$ for the control condition and $1 \mathrm{~h}$ for the practice-transfer conditions. The control condition was initially conducted as a separate experiment and, consequently, was not conducted at the same time as the other conditions.

\section{Results}

Practice session. Overall RT and PE data are reported in Table 1 for the conditions in which the subjects received prior practice with an incompatible mapping. For the data 
analyses, the practice session was divided into six blocks of 100 trials (the first block consisted only of 88 trials, since the first 12 trials were excluded as warm-up trials) for the horizontal and vertical dimensions. The main effect of block was significant for RT and PE $[F \mathrm{~s}(5,300)=$ 11.82 and 4.19, $M S_{\mathrm{e}} \mathrm{s}=999$ and 1.52, respectively; $p \mathrm{~s} \leq$ .001]. Overall, RT was longer in the first three blocks than in the last three blocks (Ms $=399,399,398,368,376$, and 379 msec for Blocks 1-6, respectively). For PE, the largest reduction was between the first and the second blocks $(M \mathrm{~s}=2.12 \%, 1.45 \%, 1.63 \%, 1.29 \%, 1.37 \%$, and $1.31 \%$ for Blocks 1-6, respectively). The only other significant effect was the block $\times$ practice dimension interaction for RT $\left[F(5,300)=2.96, M S_{\mathrm{e}}=998, p=.013\right]$, in which the vertical dimension benefited more from practice than did the horizontal dimension.

Transfer session. Mean correct RT and PE were submitted to separate 3 (practice condition: horizontalincompatible, vertical-incompatible, or control) $\times 2$ (transfer dimension: horizontal or vertical) $\times 2$ (correspondence: corresponding or noncorresponding) ANOVAs (see Table 3).

For RT, the main effect of transfer dimension was significant $\left[F(1,90)=4.85, M S_{\mathrm{e}}=7,526, p=.030\right]$, with RTs being shorter for the horizontal Simon task $(M=450 \mathrm{msec})$ than for the vertical Simon task $(M=$ $478 \mathrm{msec})$. The only other significant effect for RT was a correspondence $\times$ practice condition interaction $\left[F(2,90)=15.36, M S_{\mathrm{e}}=369, p<.001\right]$. To clarify the interaction, separate ANOVAs were performed for each practice condition. The Simon effect was significant in the control condition $\left[F(1,30)=29.51, M S_{\mathrm{e}}=315, p<\right.$ .002] and did not differ significantly for the horizontal and the vertical dimensions $[F(1,30)=1.63, p>.20]$. For the vertical-incompatible practice condition, no overall Simon effect was evident $(F<1)$, and there was no interaction with transfer dimension $(F<1)$. For the horizontal-incompatible practice condition, an overall -13-msec Simon effect was evident [i.e., longer RT for corresponding than for noncorresponding trials;
$\left.F(1,30)=6.45, M S_{\mathrm{e}}=428, p=.017\right]$ that did not interact with transfer dimension $(F<1)$.

For the PE data, the main effect of practice condition was significant $\left[F(2,90)=4.93, M S_{\mathrm{e}}=10.53, p=.009\right]$, with fewer errors committed in the control condition $(M=2.13 \%)$ than in the horizontal-incompatible $(M=$ $3.79 \%)$ and vertical-incompatible $(M=3.57 \%)$ practice conditions. Similar to the RT data, the only other significant effect for $\mathrm{PE}$ was a correspondence $\times$ practice condition interaction $\left[F(2,90)=4.84, M S_{\mathrm{e}}=5.95, p \mathrm{~s} \leq\right.$ .01]. Separate ANOVAs were performed for each practice condition to clarify this interaction. The Simon effect was significant in the control condition $[F(1,30)=11.97$, $\left.M S_{\mathrm{e}}=4.36, p<.002\right]$ and did not differ significantly for the horizontal and the vertical dimensions $(F<1)$. For the vertical-incompatible practice condition, no Simon effect was evident $(F<1)$, and there was no interaction with transfer dimension $(F<1)$. For the horizontalincompatible practice condition, no overall Simon effect was evident $(F<1)$, but correspondence interacted with transfer dimension $\left[F(1,30)=5.44, M S_{\mathrm{e}}=6.74, p=\right.$ .027]. Following horizontal practice, the vertical Simon effect was a nonsignificant $0.9 \%\left[F(1,15)=1.02, M S_{\mathrm{e}}=\right.$ $6.34, p>.30]$, whereas the horizontal Simon effect was $-2.1 \%\left[F(1,15)=5.08, M S_{\mathrm{e}}=7.13, p=.04\right]$.

Influence of the number of practice trials. To evaluate the effects of the additional practice received in the present experiment, a comparison of the practice-transfer conditions of the present experiment with the 5-min delay practice-transfer conditions in Experiment 1 was made. A 2 (practice dimension: horizontal or vertical) $\times 2$ (transfer dimension: horizontal or vertical) $\times 2$ (correspondence: corresponding or noncorresponding trials) $\times 2$ (experiment: Experiment 1 or 2) ANOVA was conducted on RT and PE. For both measures, the only significant interaction involving experiment was that of correspondence and experiment $\left[F \mathrm{~S}(1,152)=28.52\right.$ and $9.57, M S_{\mathrm{e}} \mathrm{S}=395$ and 6.53 , respectively; $p s<.002]$. This interaction reflects the fact that the Simon effect was smaller in Experiment 2 $(-7 \mathrm{msec}$ and $-0.57 \%)$ than in Experiment 1 (18 $\mathrm{msec}$

Table 3

\begin{tabular}{|c|c|c|c|c|c|c|c|c|c|c|}
\hline \multicolumn{11}{|c|}{$\begin{array}{l}\text { Mean Reaction Times (RTs) and Percentages of Errors (PEs) (With } \\
\text { Deviations) and Simon Effects for the Horizontal and Vertic } \\
\text { Simon Transfer Tasks in Experiment } 2 \text { As a Function of } \\
\text { Correspondence and Practice and Transfer Dimension }\end{array}$} \\
\hline \multirow{4}{*}{$\begin{array}{c}\text { Transfer } \\
\text { Dimension }\end{array}$} & \multicolumn{8}{|c|}{ Correspondence } & & \\
\hline & \multicolumn{4}{|c|}{ Corresponding } & \multicolumn{4}{|c|}{ Noncorresponding } & & \\
\hline & \multicolumn{2}{|c|}{ RT } & \multicolumn{2}{|c|}{$\mathrm{PE}$} & \multicolumn{2}{|c|}{ RT } & \multicolumn{2}{|c|}{$\mathrm{PE}$} & \multicolumn{2}{|c|}{ Simon Effect } \\
\hline & $M$ & $S D$ & $M$ & $S D$ & $M$ & $\overline{S D}$ & $M$ & $\overline{S D}$ & RT & $\mathrm{PE}$ \\
\hline & \multicolumn{10}{|c|}{ Control (No Prior Practice With an Incompatible Mapping) } \\
\hline Vertical & 460 & 66 & 0.57 & 0.8 & 490 & 68 & 2.85 & 2.6 & 30 & 2.28 \\
\hline Horizontal & 442 & 53 & 1.90 & 1.7 & 461 & 56 & 3.22 & 2.9 & 19 & 1.32 \\
\hline \multicolumn{11}{|c|}{ Practice With a Vertical Incompatible Mapping } \\
\hline Vertical & 461 & 49 & 3.91 & 3.9 & 460 & 52 & 3.39 & 2.2 & -1 & -0.52 \\
\hline Horizontal & 443 & 66 & 4.08 & 3.2 & 445 & 52 & 3.79 & 3.5 & 2 & -0.28 \\
\hline \multicolumn{11}{|c|}{ Practice With a Horizontal Incompatible Mapping } \\
\hline Vertical & 502 & 86 & 3.26 & 2.4 & 493 & 91 & 4.16 & 3.4 & -9 & 0.90 \\
\hline Horizontal & 464 & 53 & 4.49 & 4.2 & 447 & 43 & 2.37 & 1.8 & -17 & -2.12 \\
\hline
\end{tabular}


and $1.29 \%$ ), showing a greater influence of the practice mapping after 600 trials of practice than after 72 trials. Otherwise, the patterns of results across the two experiments were similar.

\section{Discussion}

For the practice session, overall RT and PE decreased with practice, replicating previous studies (e.g., Dutta \& Proctor, 1992). Furthermore, the vertical dimension benefited more from practice than did the horizontal dimension. For the horizontal-horizontal practice-transfer condition, the increased practice was sufficient to reverse the overall horizontal Simon effect in the transfer session. The reverse Simon effect for RT in this experiment was significantly different from the nonsignificant Simon effect obtained after only 72 trials of practice in Experiment 1 $\left[F(1,38)=7.63, M S_{\mathrm{e}}=261, p=.009\right]$. This larger transfer effect to the horizontal Simon task is in agreement with the view that the noncorresponding $\mathrm{S}-\mathrm{R}$ associations learned in practice were strengthened.

Unlike in Experiment 1, though, the transfer effect for the horizontal Simon task occurred between dimensions, as well as within the same dimension. A between-dimension transfer effect cannot be attributed to the strengthening of specific S-R associations. Thus, the larger transfer effect in Experiment 2 is likely due to subjects' acquiring a respond-opposite procedure during the practice task, which was then transferred to the Simon task. Although the RT data showed no significant difference between the magnitude of the transfer effect within and between dimensions, the error data showed that the transfer of noncorresponding $\mathrm{S}-\mathrm{R}$ associations to the horizontal Simon task was larger when the practice dimension was also horizontal. This finding suggests that for the horizontalhorizontal condition, there may also be a benefit of specific $\mathrm{S}-\mathrm{R}$ associations.

The vertical Simon effect was also eliminated after 600 trials of practice with an incompatible mapping. The fact that the vertical Simon effect was not eliminated after 72 trials of practice in Experiment 1 indicates that the noncorresponding $\mathrm{S}-\mathrm{R}$ associations were more difficult to acquire and/or apply with the vertical dimension. This difficulty may be related to differences in how the horizontal and the vertical dimensions are processed (see Wiegand \& Wascher, 2005) or to the fact that the horizontal dimension also benefits from the spatial coding provided by the left and right hands (see Vu \& Proctor, 2001). More important, the finding of a between-dimension transfer effect for the vertical Simon task implies that the subjects learned a respond-opposite procedure during the practice task that was the basis for the transfer. Although the strengthening of specific $\mathrm{S}-\mathrm{R}$ associations may have been the cause of the transfer effect for the vertical-practice-verticaltransfer condition, the similar magnitudes of the withinand between-dimension transfer effects suggests that they are due to the same mechanism, that of the respondopposite procedure.

The between-experiment analysis showed that the overall Simon effect was countered more by 600 trials of practice with an incompatible spatial mapping than with 72 trials. The finding that experiment did not interact significantly with practice dimension and transfer dimension, alone or in combination, suggests that the influence of additional practice is similar across all four practice-transfer conditions. That the horizontal-horizontal practicetransfer condition showed larger effects in the PE data and a trend in that direction for the RT data suggests that for the horizontal Simon task, the specific associations that affect performance in that condition after 72 practice trials may still be contributing, in addition to the rule-based procedure after 600 practice trials. Although the amount of practice is the most likely source of the different results for Experiments 1 and 2, it should be noted that, as with most studies of practice, the subjects who received more practice also spent a longer total time on the task.

\section{GENERAL DISCUSSION}

The present study confirmed that when the amount of practice with an incompatible mapping was small (e.g., 72 practice trials), the horizontal Simon effect was eliminated when the practice task involved an incompatible mapping of left-right locations mapped to keypresses. Furthermore, the influence of the incompatible mapping on the Simon transfer task was just as strong 1 week after the practice session as it was after only $5 \mathrm{~min}$. The finding that the transfer effect is evident after a 1-week delay suggests that for the horizontal dimension, the noncorresponding $\mathrm{S}-\mathrm{R}$ associations learned during the practice session are reinstated by the task context, even though stimulus location is nominally irrelevant in the Simon transfer task. Healy et al. (2005) also found evidence for procedural reinstatement after a 1-week delay in a perceptual-motor task. In their study, subjects learned to control a mouse that was programmed to make left-right reversals (e.g., leftward movement of the mouse resulted in rightward movement of the cursor), top-bottom reversals, or both types of reversal. This learning benefited performance of the same task a week later only when the mouse was programmed the same as in practice. No transfer was evident when a mouse programmed differently was used (e.g., learning with a mouse that had both left-right and top-bottom reversals did not improve performance when tested with a mouse that produced only left-right reversals or only top-bottom reversals).

Although, in Experiment 1, the horizontal Simon effect was eliminated after practice with 72 trials of a horizontal-incompatible mapping, the vertical Simon effect remained evident after the subjects performed 72 trials with an incompatible mapping of vertically arrayed stimuli and responses. The lack of a generalization of the within-dimension transfer effect to the vertical dimension may be due to the horizontal and the vertical Simon effects having different processing bases (Wiegand \& Wascher, $2005)$. With the 72 trials of practice, there also was no evidence of the prior incompatible mapping on one spatial dimension transferring to the other dimension in the transfer Simon task. The absence of between-dimension transfer effects provides no evidence that a general respondopposite procedure was acquired. 
When the number of practice trials was increased from 72 to 600 in Experiment 2, large transfer effects were evident, both within and between spatial dimensions. Thus, extended practice with an incompatible location mapping can strengthen the task-defined, noncorresponding S-R associations sufficiently for them to remain active and override the benefits of the long-term, corresponding S-R associations in the transfer session. Although the withindimension transfer effects may be a result of learning to make a specific response to a specific stimulus location, the between-dimension transfer effects can be attributed only to rule-based procedures (see Rosenbloom, 1986). Because the increase in size of the transfer effect after 600 practice trials, as compared with 72 practice trials, was just as large for all four practice-transfer conditions, within and between dimensions, it is likely that the entire effect is due to acquisition of rule-based procedures. However, the finding that the horizontal-horizontal practicetransfer condition still tends to yield larger transfer effects than do the other conditions at the long delay suggests that for that condition, there may be a specific component to the transfer effect, in addition to the rule-based one.

Hedge and Marsh (1975) proposed that when response keys are colored and the mapping of stimulus color to response key color is incompatible (i.e., press the green key if the stimulus is red and the red key if the stimulus is green), a respond-opposite rule for color is also applied to the location dimension. They based their proposal on the Simon effect's being reversed in this situation, for which the relevant dimension was mapped incompatibly. Whereas Hedge and Marsh proposed that a currently relevant respond-opposite rule is unintentionally applied to the spatial dimension, the present findings suggest that a respond-opposite procedure that was previously relevant for stimulus location continues to be applied when it is no longer relevant. Both proposals imply that general response selection rules can be formed. The contribution of the present study is to show that once these rules are proceduralized, they will be transferred automatically to related tasks for which the rule is no longer appropriate.

Although the elimination of the Simon effect after prior practice with an incompatible location mapping can be discussed in terms of the strength and type of S-R associations that are formed and transferred, another possible reason why the Simon effect is eliminated in the transfer session is that there is an increase in cognitive control following performance with an incompatible mapping of S-R locations (see, e.g., Botvinick, Braver, Barch, Carter, \& Cohen, 2001). Botvinick, Cohen, and Carter (2004) reviewed evidence that the anterior cingulate cortex is involved in conflict monitoring (it is activated on incongruent trials in Stroop color-naming tasks [Stroop, 1935] and Eriksen flanker tasks [Eriksen \& Eriksen, 1974]) and that its activation is associated with increased activity in brain regions, such as the prefrontal cortex, that are associated with cognitive control. Increased cognitive control following practice with an incompatible mapping could lead to the elimination of the Simon effect because attention is focused more on the relevant color dimension, reducing the influence of the irrelevant location dimension.
The increased-cognitive-control hypothesis can account for the finding that the horizontal Simon effect is eliminated after 72 trials are performed with an incompatible mapping of left-right locations at the 5-min delay. However, as with the reactivation of specific $\mathrm{S}-\mathrm{R}$ associations hypothesis, it does not account for why the vertical Simon effect was unaffected by previous performance with 72 trials of an incompatible mapping of top-bottom locations. There is a possibility, though, that the increased cognitive control eliminates only the transient benefit found for the horizontal Simon task (see Wiegand \& Wascher, 2005). The hypothesis of increased cognitive control also seems to be more plausible at the 5-min delay than at the 1 -week delay, unless there is a reactivation of the conflicting associations when the subjects return to the lab a week later. Moreover, the hypothesis cannot account for why the horizontal Simon effect reversed when the number of practice trials with an incompatible mapping was increased in Experiment 2, without the addition of the learning of a respond-opposite procedure that was applied in the transfer session. Thus, it seems that the role of increased cognitive control occurs in addition to the transfer of S-R associations discussed earlier. In future studies, the role of increased cognitive control following conflict detection should be directly compared with that of the transfer of specific or rule-based S-R associations in eliminating the Simon effect.

In summary, a limited amount of practice can produce new task-defined spatial S-R associations that persist at a sufficient strength to override the long-term associations between corresponding locations when the S-R sets vary along the horizontal dimension in the practice and transfer sessions. With limited practice, the transfer effect is not evident when the S-R sets vary along the vertical dimension in the practice and transfer sessions or when the dimension in the practice session is orthogonal to that in the transfer session. With more practice on the spatial task, subjects acquire a general response selection procedurerespond opposite to the stimulus location - that transfers across dimensions to a subsequent task for which stimulus location is nominally irrelevant.

\section{AUTHOR NOTE}

I thank the following individuals for assistance in conducting the experiments: Rachael Anderson, Abhilasha Bhargav, Rob Janik, Leigh Lytle, Srikanth Ramachandran, Kathy Schnirring, Amy Smith, Amanda Smith, Zac Smith, Bik-Lam Tai, and Kerry Thorne. I also thank Veronica Dark, Michael Kane, Sylvan Kornblum, James Nairne, David Pick, Wolfgang Prinz, Robert Proctor, Carlo Umiltà, and Howard Zelaznik for helpful comments on a previous version of the manuscript. Correspondence regarding this article should be sent to K.-P. L. Vu, Department of Psychology, California State University, 1250 Bellflower Blvd., Long Beach, CA 90840 (e-mail: kvu8@csulb.edu).

\section{REFERENCES}

BARBER, P., \& O'LeARY, M. (1997). The relevance of salience: Towards an activation account of irrelevant stimulus-response compatibility effects. In B. Hommel \& W. Prinz (Eds.), Theoretical issues in stimulusresponse compatibility (pp. 135-172). Amsterdam: North-Holland.

Botvinick, M. M., Braver, T. S., Barch, D. M., Carter, C. S., \& Cohen, J. D. (2001). Conflict monitoring and cognitive control. Psychological Review, 108, 624-652. 
Botvinick, M. M., Cohen, J. D., \& Carter, C. S. (2004). Conflict monitoring and anterior cingulate cortex: An update. Trends in Cognitive Sciences, 8, 539-546.

Donders, F. C. (1969). On the speed of mental processes. In W. G. Koster (Ed.), Attention and performance II (pp. 412-431). Amsterdam: North-Holland. (Original work published 1868)

Duncan, J. (1977). Response selection rules in spatial choice reaction tasks. In S. Dornic (Ed.), Attention and performance VI (pp. 49-61). Hillsdale, NJ: Erlbaum.

Dutta, A., \& Proctor, R. W. (1992). Persistence of stimulus-response compatibility effects with extended practice. Journal of Experimental Psychology: Learning, Memory, \& Cognition, 18, 801-809.

EriKsen, B. A., \& ERIKSEN, C. W. (1974). Effects of noise letters upon the identification of a target letter in a nonsearch task. Perception \& Psychophysics, 16, 143-149.

FitTs, P. M. (1964). Perceptual-motor skill learning. In A. W. Melton (Ed.), Categories of human learning (pp. 243-285). New York: Academic Press.

Healy, A. F., Wohldmann, E. L., \& Bourne, L. E., JR. (2005). The procedural reinstatement principle: Studies on training, retention, and transfer. In A. F. Healy (Ed.), Experimental cognitive psychology and its applications (pp. 59-71). Washington, DC: American Psychological Association.

Hedge, A., \& Marsh, N. W. A. (1975). The effect of irrelevant spatial correspondences on two-choice response time. Acta Psychologica, 39, 427-439.

Hommel, B. (1997). Interactions between stimulus-stimulus congruence and stimulus-response compatibility. Psychological Research, 59, 248-260.

Kornblum, S., Hasbroucq, T., \& Osman, A. (1990). Dimensional overlap: Cognitive basis for stimulus-response compatibility-a model and taxonomy. Psychological Review, 97, 253-270.

Logan, G. D. (1988). Toward an instance theory of automatization. Psychological Review, 95, 492-527.

Lu, C.-H., \& Proctor, R. W. (1995). The influence of irrelevant location information on performance: A review of the Simon and spatial Stroop effects. Psychonomic Bulletin \& Review, 2, 174-207.

Nicoletti, R., Anzola, G. P., Luppino, G., Rizzolatti, G., \& UMILTÀ, C. (1982). Spatial compatibility effects on the same side of the body midline. Journal of Experimental Psychology: Human Perception \& Performance, 8, 644-673.

Nicoletti, R., \& Umiltà, C. (1985). Responding with hand and foot: The right/left prevalence in spatial compatibility is still present. Perception \& Psychophysics, 38, 211-216.

Pashler, H., \& BaYlis, G. C. (1991). Procedural learning: Locus of practice effects in speeded choice tasks. Journal of Experimental Psychology: Learning, Memory, \& Cognition, 17, 20-32.

Proctor, R. W., \& Dutta, A. (1995). Acquisition and transfer of response-selection skill. In A. F. Healy \& L. E. Bourne, Jr. (Eds.), Learning and memory of knowledge and skills: Durability and specificity (pp. 300-319). Thousand Oaks, CA: Sage.

Proctor, R. W., \& Lu, C.-H. (1999). Processing irrelevant location information: Practice and transfer effects in choice-reaction tasks. Memory \& Cognition, 27, 63-77.

Proctor, R. W., \& Reeve, T. G. (1985). Compatibility effects in the assignment of symbolic stimuli to discrete finger response. Journal of Experimental Psychology: Human Perception \& Performance, 11, 623-639.

Proctor, R. W., \& Reeve, T. G. (1986). Salient-feature coding operations in spatial precuing tasks. Journal of Experimental Psychology: Human Perception \& Performance, 12, 277-285.
Proctor, R. W., \& Vu, K.-P. L. (2002). Eliminating, magnifying, and reversing spatial compatibility effects with mixed location-relevant and irrelevant trials. In W. Prinz \& B. Hommel (Eds.), Common mechanisms in perception and action: Attention and performance XIX (pp. 443-473). Oxford: Oxford University Press.

Proctor, R. W., \& Vu, K.-P. L. (2006). Stimulus-response compatibility: Data, theory, and application. Boca Raton, FL: CRC Press.

Proctor, R. W., Vu, K.-P. L., \& Nicoletti, R. (2003). Does right-left prevalence occur for the Simon effect? Perception \& Psychophysics, 65, 1318-1329.

RosEnBLOOM, P. S. (1986). The chunking of goal hierarchies: A model of stimulus-response compatibility and practice. In J. E. Laird, P. S. Rosenbloom, \& A. Newell (Eds.), Universal subgoaling and chunking: The automatic generation and learning of goal hierarchies (pp. 133-282). Boston: Kluwer.

Roswarski, T. E., \& Proctor, R. W. (2000). Auditory stimulusresponse compatibility: Is there a contribution of stimulus-hand correspondence? Psychological Research, 63, 148-158.

Simon, J. R. (1990). The effects of an irrelevant directional cue on human information processing. In R. W. Proctor \& T. G. Reeve (Eds.), Stimulus-response compatibility: An integrated perspective (pp. 3186). Amsterdam: North-Holland.

Simon, J. R., \& Rudell, A. P. (1967). Auditory S-R compatibility: The effect of an irrelevant cue on information processing. Journal of Applied Psychology, 51, 300-304.

Stoffer, T. H., \& Umiltà, C. (1997). Spatial stimulus coding and the focus of attention in S-R compatibility and the Simon effect. In B. Hommel \& W. Prinz (Eds.), Theoretical issues in stimulus-response compatibility (pp. 181-208). Amsterdam: North-Holland.

Stroop, J. R. (1935). Studies of interference in serial verbal reactions. Journal of Experimental Psychology, 18, 643-662.

Tagliabue, M., Zorzi, M., \& Umiltà, C. (2002). Cross-modal re-mapping influences the Simon effect. Memory \& Cognition, 30, 18-23.

Tagliabue, M., Zorzi, M., Umiltà, C., \& Bassignani, F. (2000). The role of LTM links and STM links in the Simon effect. Journal of Experimental Psychology: Human Perception \& Performance, 26, 648-670.

Vu, K.-P. L., Pellicano, A., \& Proctor, R. W. (2005). No overall right-left prevalence for horizontal and vertical Simon effects. Perception \& Psychophysics, 67, 929-938.

Vu, K.-P. L., \& Proctor, R. W. (2001). Determinants of right-left and top-bottom prevalence for two-dimensional spatial compatibility. Journal of Experimental Psychology: Human Perception \& Performance, 27, 813-828.

Vu, K.-P. L., Proctor, R. W., \& Pick, D. F. (2000). Vertical versus horizontal spatial compatibility: Right-left prevalence with bimanual responses. Psychological Research, 64, 25-40.

Vu, K.-P. L., Proctor, R. W., \& Urcuioli, P. (2003). Transfer effects of incompatible location-relevant mappings on a subsequent visual or auditory Simon task. Memory \& Cognition, 31, 1146-1152.

Wiegand, K., \& WAScher, E. (2005). Dynamic aspects of stimulusresponse correspondence: Evidence for two mechanisms involved in the Simon effect. Journal of Experimental Psychology: Human Perception \& Performance, 31, 453-464.

ZorZI, M., \& UMiLtì, C. (1995). A computational model of the Simon effect. Psychological Research, 58, 193-205.

(Manuscript received December 15, 2005; revision accepted for publication April 24, 2006.) 